\title{
NEFRECTOMÍA LAPAROSCÓPICA CON UN SOLO TROCAR.
}

\author{
Eduardo Sánchez de Badajoz y Adolfo Jiménez Garrido.
}

Área de Conocimiento de Urología. Facultad de Medicina. Universidad de Málaga. Málaga. España.

Resumen.- OBJETIVO: Normalmente la nefrectomía asistida se realiza con dos o tres trócares junto con una mini-incisión para extraer la pieza operatoria. Nosotros hemos comprobado que se puede disminuir significativamente la agresión quirúrgica, realizándola por vía extraperitoneal y con un solo trocar.

MÉTODOS: Se empieza haciendo una mini-incisión oblicua muy baja, próxima al pubis. Ponemos el único trocar sobre la cresta ilíaca. Una vez que tenemos liberado el riñón, sujetamos el pedículo, sin disecarlo, entre el dedo pulgar y el dedo medio, acto seguido, sin mover la mano interna, sacamos la óptica y metemos la grapadora. Con el dedo índice, a ciegas, comprobamos que el instrumento está en posición correcta y lo disparamos. Por último ligamos el uréter de igual manera. Hemos hecho un total de 74 nefrectomías.
Eduardo Sánchez de Badajoz Calle Strachan, 4 - 2 piso 29015 Málaga. (España) edusaba@telefonica.net Trabajo recibido: 3 de enero 2006
RESULTADOS: El tiempo operatorio medio fue de 67, 12 minutos. En un paciente, se formó un hematoma retroperitoneal, que se drenó por la misma vía de abordaje, sin incidencias. Una paciente nos hizo una oclusión intestinal; en la reintervención, comprobamos que había un asa de intestino, que se había metido por una brecha de peritoneo; el postoperatorio fue bueno. Hubo un éxitus en una enferma de 89 años con una pionefrosis y un absceso retroperitoneal; la operación se hizo sin problemas, sin embargo la enferma murió a los 5 días, posiblemente de un shock séptico. En el resto de los casos no hubo complicaciones significativas.

CONCLUSIONES: El uso de un solo trocar disminuye drásticamente la agresión operatoria. La ligadura en bloque y a ciegas del pedículo, ahorra un tiempo muy valioso, no supone ningún riesgo añadido y la posibilidad de fístula arteriovenosa es remota, porque la grapadora deja la arteria cerca de la vena pero separada de la misma. El gasto de material es mínimo, puesto que sólo se requiere un trocar y la grapadora. Por todo ello creemos que debería plantearse incluso su utilización en la extracción de donante vivo.

Palabras clave: Nefrectomía. Laparoscopia. Manualmente asistida. Un solo trocar.

Summary.- OBJECTIVES: The usual laparoscopic hand assisted nephrectomy procedure requires either two or three trocars and a small incision to extract the organ. Our experience reveals that surgical invasion is significantly less when we use only one trocar and a retroperitoneal approach.

METHODS: We start with a small low oblique incision close to the pubis and insert a trocar over the iliac crest. When the kidney is free we grasp the undissected vascular pedicle between thumb and middle finger and without moving the inserted hand, we remove the tele- 
scope and introduce the stapler. The index finger is used blind to check that the stapler is correctly positioned before triggering. Finally, we divide the ureter in the same way. We have carried out 74 nephrectomies with this procedure

RESULTS: The mean operative time was 67.12 minutes. A retroperitoneal haematoma occurred in one patient and was drained by the same approach without further incident. One patient had an intestinal occlusion that in the reoperation was found to be caused by a loop of intestine trapped in a peritoneal tear: the postoperative was satisfactory. We lost one patient: an 89-year-old lady with a pyonephrosis and a retroperitoneal abscess. The operation was uneventful, but the patient died after five days; possibly because of septic shock. The other patients had no significant complications.

CONCLUSIONS: The use of only one trocar markedly reduced operative invasion. The blind division en-bloc of the pedicle saves valuable time and does not offer any additional risk. The possibility of an arterio-venous fistula is remote because the stapler leaves the artery close to the vein, but separated from it. Cost is minimal because the surgeon only needs one trocar and a stapler. Consequently, we believe that this procedure with only one trocar could also be safely adopted for living donor nephrectomy.

Keywords: Nephrectomy. Laparoscopy. Hand assisted. Only one trocar. Only one port.

\section{INTRODUCCIÓN}

En lo que va de siglo XXI, la cirugía laparoscópica urológica ha llegado a tal punto, que da la impresión de que todo está ya hecho y que difícilmente se puede avanzar más. Aparentemente todo está ya inventado. Buena prueba de ello es que casi todo el mundo se limita a hacer lo mismo prácticamente, sin aportar nada nuevo. Sin embargo estamos convencidos, de que a corto plazo, queda mucho por hacer, mucho por descubrir, más de lo que nos podemos imaginar.

Muchos de nosotros en alguna ocasión, al ver una nueva técnica publicada, nos hemos preguntado ¿̇ por qué no se nos ha ocurrido a nosotros? $O$ lo que es peor ¡Eso ya lo habíamos pensado! $O$ más grave todavía ¡Esa técnica que publican ahora como novedad mundial, hace años que la llevamos haciendo nosotros! Pero sencillamente nos tenemos que resignar por no haberla comunicado; eso que habíamos considerado una obviedad, resulta que ahora es una primicia.
Desde hace más de una década venimos realizando sistemáticamente nefrectomías asistidas, técnica que sin duda supuso una revolución en la laparoscopia urológica $(1,2)$. Sin embargo, casi desde el principio nos preguntamos si era posible transformar esta intervención en un procedimiento aún menos invasivo. La respuesta es que sí, que es posible y esto es lo que nos ha decidido a escribir este artículo. Normalmente esta técnica requiere el uso de dos o tres trócares más una mini-incisión para extraer el órgano. Nosotros hemos comprobado que es posible realizarla con toda garantía con un único trocar, tal y como vamos a describir a continuación.

\section{MATERIAL Y MÉTODOS}

Ponemos al paciente en posición de lumbotomía. El monitor y el resto de la torre de laparoscopia lo situamos a la cabecera de la mesa, junto al aparato de anestesia. El cirujano se pone en el lado ventral del paciente, el ayudante va a la espalda del enfermo; es decir, justo al contrario de lo que se hace en una lumbotomía convencional (3).

Empezamos haciendo una incisión oblicua, muy baja, de 7 u 8 centímetros, que termina a unos 3 centímetros del pubis. Llegamos a las fascias, las incidimos y en el extremo medial de la incisión nos vamos a encontrar con el músculo recto abdominal, del que traccionamos. A menudo aparecen los epigástricos, que también rechazamos medialmente. Una vez que hemos llegado al peritoneo, sin abrirlo lo despegamos del resto de la pared en dirección a la cresta ilíaca. Esta maniobra la hacemos primero con el dedo índice hasta que conseguimos introducir el resto de la mano, siempre sin romper el peritoneo. Es muy importante lubricar la mano con una crema antibiótica, Furacin ${ }^{\circledR}$ por ejemplo, lo que nos permitirá introducirla por una incisión menor de lo que imaginamos (4). Los dispositivos diseñados para que no se escape el gas, sencillamente no tienen utilidad alguna y además son muy caros.

Partiendo de la base de que todo cirujano que se precie tiene que ser ambidiestro y que no hay una mano dominante, sino que ambas manos tienen que ser igual de dominantes, recomendamos empezar por introducir la mano izquierda, si estamos operando el riñón derecho y a la inversa. De cualquier forma, a lo largo de la intervención podemos introducir indistintamente una $u$ otra mano según nos interese.

De entrada, nada más empezar, con la mano introducida, vamos a despegar toda la pared lumbar posterior de la fascia de Gerota hasta llegar al polo 
superior del riñón. Después introducimos el único trocar a unos dos centímetros por encima de la cresta ilíaca, en el sitio que nos queda en situación más elevada y que corresponde aproximadamente a la línea axilar media. Después metemos la óptica y el gas. Con la mano interna, siguiendo el plano ya iniciado, continuamos despegando la fascia de Gerota por la porción superior junto al diafragma y por la cara anterior, siguiendo el plano ya iniciado, liberándola suavemente del peritoneo sin romperlo. Llega un momento en que toda la pieza estará aislada excepto el pedículo y el uréter. La situación del trocar y de la óptica nos proporcionan una buena perspectiva del pedículo, que sujetaremos entre nuestro dedo pulgar y dedo medio (Figura 1), que no vamos a disecar. Con frecuencia la maniobra de Kocher, es decir voltear el riñón medialmente, nos puede hacer mejorar la visión del pedículo.

Seguidamente, una vez que tenemos enfilado y "a tiro" el pedículo, viene la maniobra decisiva, para la cual dejamos la mano inmóvil, sacamos la óptica, metemos la grapadora-cortadora, con carga vascular, hasta incluir todo el pedículo (Figura 2). Con el dedo índice, sin visión, comprobamos que el instrumento está en posición correcta, metido a fondo y que no incluye nada que no deba. Y a continuación sencillamente disparamos a ciegas pero con todas

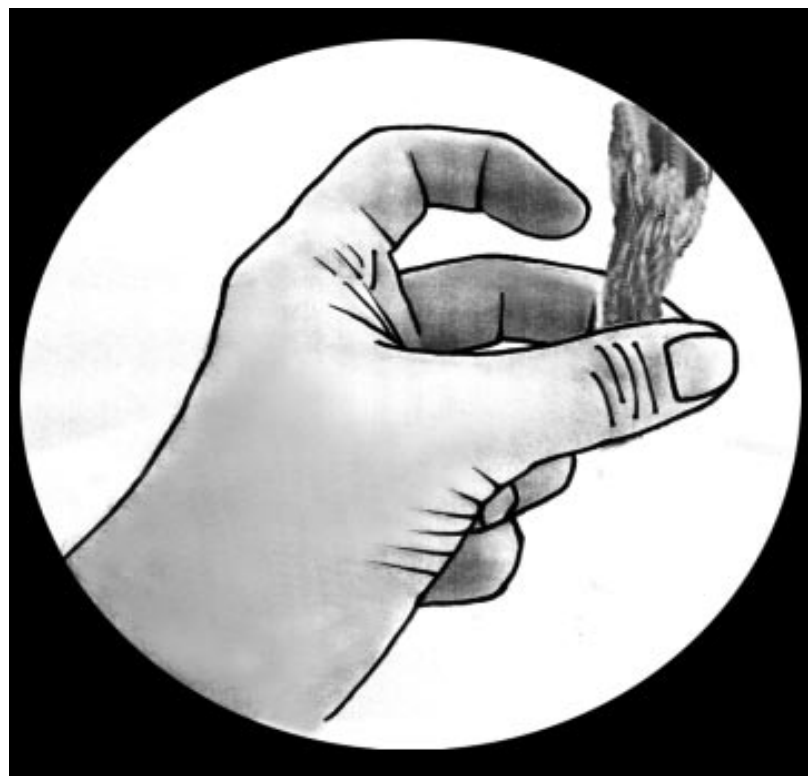

FIGURA 1. Una vez que se ha liberado totalmente el riñón, se sujeta el pedículo entre los dedos pulgar y medio. las garantías (Figura 3). A veces, si nos queda alguna brida, puede ser necesario un segundo disparo, que haremos de igual forma. Por último, con el mismo instrumento y también a ciegas, disparamos sobre el uréter, con lo cual la pieza se nos queda libre en el retroperitoneo. Ni que decir tiene que si hay vasos polares se graparán independientemente.

Para extraer el órgano, tal y como ya hemos descrito, introduciremos en el abdomen una bolsa recolectora de orina recortada por el lado del tubo. Sin embargo a menudo ocurre que la pieza no cabe en la bolsa, entonces utilizamos una bolsa de tres litros de capacidad como son las de glicina, recortada y abierta igualmente por el borde del tubo. La bolsa en el momento de introducirla, la ponemos en la mano vuelta como un calcetín, lo que nos permitirá coger directa y fácilmente el riñón. Por último sacamos al exterior los bordes de la bolsa, los sujetamos con pinzas de Kocher, fragmentamos la pieza con el dedo, hasta triturarla y finalmente la extraemos.

Hasta la fecha hemos operado un total de 74 nefrectomías asistidas con un solo trocar, en pacientes de edades comprendidas entre los 37 y 89 años, de las cuales 56 se han realizado por patología neoplásica y 18 se han hecho por patología no tumoral.

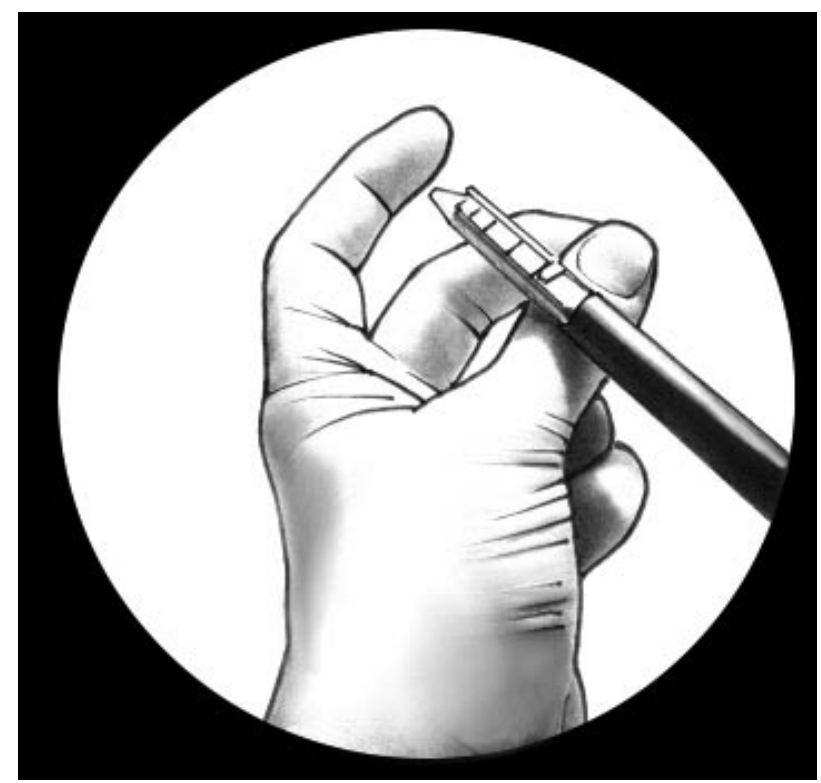

FIGURA 2. Con la grapadora-cortadora se incluye el pedículo en bloque (no dibujado). Con el dedo índice y a ciegas, se comprueba que el instrumento no incluye nada que no deba y a continuación se dis-para. 


\section{RESULTADOS}

En los 74 casos operados con la técnica descrita, el tiempo operatorio medio, de piel a piel, fue de 67,12 minutos, teniendo en cuenta que en los primeros casos el tiempo era significativamente mayor por la curva de aprendizaje y por el hecho que supone que el momento decisivo de la operación se haga a ciegas.

Complicaciones intraoperatorias no ha habido ninguna y no ha habido ningún caso en que tuviéramos que reconvertir.

En cuanto al postoperatorio inmediato, en un caso, a las 7 u 8 horas de la intervención, el paciente se descompensó hemodinámicamente y se anemizó. Se hizo una TC y se comprobó que había un hematoma retroperitoneal y a continuación se llevó el enfermo a quirófano. Por la misma vía de abordaje, se vació el hematoma y no se encontró ningún vaso sangrante, lo que demostró que se trataba de una hemorragia en sábana. Algo similar a lo que ocurre cuando una próstata nos sangra, que volvemos a meter el resector, vaciamos los coágulos y no aparece ningún punto sangrante. Este paciente se fue de alta al tercer día de la intervención. Este caso nos enseñó mucho, porque pudimos demostrar cómo a través del único trocar y la mini-incisión, se puede resolver un problema grave como es un hematoma retroperitoneal importante.

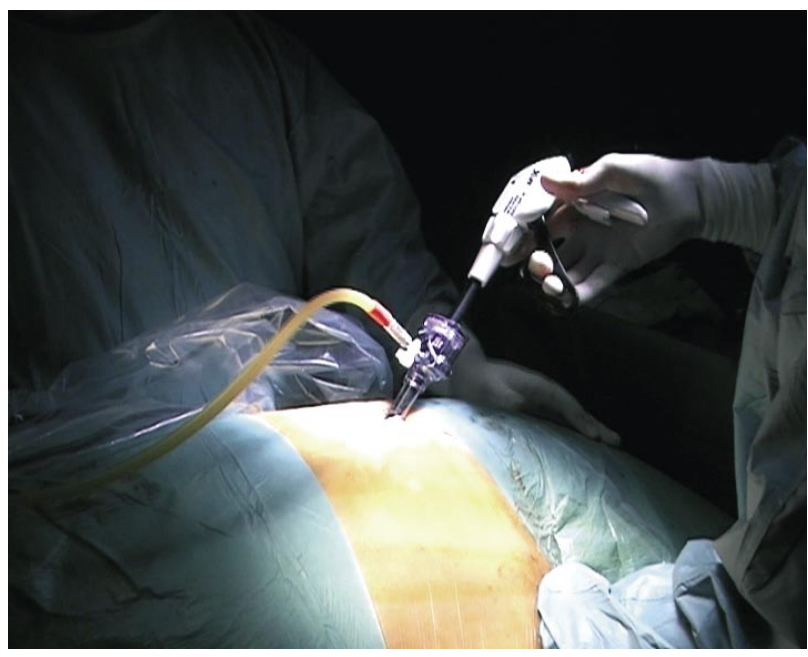

FIGURA 3. El trocar único si sitúa justo por encima de la cresta ilíaca y la mini-incisión se hace junto al pubis. Se acaba de sacar la óptica y en este momento se está disparando la grapadora sobre el pedí-culo, maniobra que se controla con la mano izquierda.
En otro caso, en el postoperatorio tardío hubo una infección de la pared abdominal en una paciente muy obesa, que requirió tres semanas de hospitalización. En cuanto a la mini-incisión ha habido dos casos de hernia de la misma.

En una paciente, a la semana de la intervención en el lado izquierdo, nos hizo una oclusión intestinal. La reoperamos a través de una laparotomía media supraumbilical y encontramos que el peritoneo tenía una brecha, por donde se había metido un asa de yeyuno, comprobamos que el asa tenía buen aspecto y solucionamos el problema no cerrando el orificio peritoneal, sino ampliándolo para que no se volviera a estrangular ningún asa. Desde esa fecha, si durante la laparoscopia abrimos sin querer el peritoneo, lo que hacemos es dejar una amplia incisión peritoneal.

Por último, dentro de las complicaciones tardías, tuvimos un éxitus en una paciente de 89 años, con una pionefrosis y un flemón perinefrítico izquierdo. $\mathrm{Ni}$ que decir tiene que antes de la intervención, se le puso un catéter percutáneo, que no fue suficiente para drenar el absceso. La intervención se hizo con la técnica descrita sin ninguna dificultad, el tiempo operatorio fue de 70 minutos y no rompimos el peritoneo, sin embargo la paciente murió a los cinco días, muy probablemente de un shock séptico. La pieza quirúrgica pesó 496 gramos y el diagnostico patológico vino con sorpresa, ya que fue un carcinoma sarcomatoide; sin duda origen de la obstrucción, de la pionefrosis y del absceso.

\section{DISCUSIÓN}

Ante todo queremos recordar que por vía laparoscópica convencional, difícilmente se tarda menos de tres horas en las manos más expertas y así todo hay muchos riñones inoperables, que sí lo serían por vía asistida, además en un tiempo considerablemente más corto, más breve incluso que por vía abierta convencional (5).

Si la masa renal es grande y sobre todo si el paciente además es obeso, la pieza quirúrgica a extraer con toda su grasa, supera fácilmente el kilo de peso, lo que obliga a hacer una incisión al final de la intervención. Y por tanto es absurdo estar 3 ó 4 horas haciendo la nefrectomía laparoscópica a través de 4 ó 5 trócares, y al final hacer una brecha subcostal para sacar el órgano, por donde curiosamente se podría haber metido la mano al principio.

Sin embargo la crítica que muchos harán a esta técnica, es que a estas alturas no se puede ligar 
un pedículo renal en bloque, entre otras cosas porque se puede producir una fístula arteriovenosa. La respuesta en nuestra opinión, es que estamos totalmente de acuerdo en que en el siglo XXI la ligadura en bloque del pedículo, salvo excepciones, es inadmisible y menos dando un punto por transfixión, como hacían muchos cirujanos sistemáticamente hasta no hace mucho tiempo. De hecho nosotros, cuando hacíamos la nefrectomía convencional, salvo contadísimas excepciones, siempre podíamos identificar la arteria y ligarla antes que la vena. Pero si lo pensamos bien, la técnica que ahora describimos, dista mucho de ser una ligadura en bloque propiamente dicha, porque la grapadora no deja un muñón apretado, comprimido y estrujado, sino que sencillamente grapa y corta linealmente primero la vena y después la arteria, es decir se dejan en cierto modo separadas una de la otra, lo que hace que la posibilidad de fístula sea remota. Pero es que además ahorramos un tiempo valiosísimo, que es el que necesitaríamos para disecar el pedículo. Sin embargo, en nuestra experiencia, a menudo nos ocurre que con la mano, casi sin quererlo, separamos la arteria de la vena rápidamente, lo que nos permite ligarlas por separado, tal y como hemos descrito, primero la arteria y luego la vena. Pero es que además, la fístula arteriovenosa es una complicación tan rara, que nosotros no hemos visto nunca, y creemos que serán pocos los urólogos que se hayan encontrado alguna en su vida.

Alguien puede cuestionar si realmente merece la pena ahorrar uno o dos trócares. Sin duda que sí que merece la pena evitarle al paciente una o dos punciones. No olvidemos, que una grapadora requiere un trocar de 12 milímetros, lo cual es una auténtica "puñalada" y si podemos limitarla a una en vez de dos o tres, disminuiremos drásticamente la agresión operatoria, y todo lo que ello conlleva en cuanto a la necesidad de analgésicos postoperatorios y el tiempo de recuperación. Pero es que además, el poner un solo trocar acorta significativamente el tiempo operatorio, porque no olvidemos, que una vez terminada la operación, hay que comprobar detenidamente si las heridas dejadas por los trócares sangran y además hay que cerrarlas en dos planos y bien.

En un caso operamos un paciente de 83 años, cardiópata e hipocoagulado con dicumarínicos, con un gran tumor izquierdo y un trombo neoplásico en la vena renal que no llegaba a la cava. Lo operamos con la técnica descrita. Por ser el lado izquierdo, la vena era larga, la palpamos hasta llegar a donde terminaba el trombo y a continuación ligamos el pedículo de la forma descrita. El tiempo operatorio fue de 70 minutos, la pieza operatoria pesó 400 gramos y al tercer día el enfermo se fue de alta.
En cuanto a la cuestión de que si esta cirugía reúne los requisitos para ser oncológicamente correcta, estamos convencidos de que sí que lo es, además permite operar a pacientes mayores y de alto riesgo, que hasta ahora eran desahuciados. De todas formas no queremos responder a esta pregunta y dejar que sea el tiempo quién lo decida.

En las pionefrosis accedemos por el plano de abordaje del cáncer, es decir alrededor de la fascia de Gerota, con lo cual nos alejamos lo más posible de la zona inflamatoria y llegamos más fácilmente y con más seguridad al pedículo. No olvidemos que vamos por vía retroperitoneal, que es la indicada en estos casos, ya que el abordaje transperitoneal en un riñón inflamatorio, como algunos defienden, para nosotros es una contraindicación absoluta.

En cuanto a la incisión para introducir la mano y sacar la pieza, debe ser lo más baja posible porque es menos dolorosa, porque no Interfiere con la respiración y es más estética. No tiene sentido hacer una minilumbotomía, porque además nos exponemos a lesionar los abdominogenitales y dejar un hemiabdomen hipotónico e hipoestésico, a veces de por vida.

Por otra parte, creemos que esta técnica podría ser también utilizada para la extracción de donante vivo, ya que nos permite dejar una arteria y una vena de longitud más que suficiente. No cabe duda de que al disminuir drásticamente el tiempo operatorio y evitarle al donante sano una o dos gruesas perforaciones en la pared abdominal, aumentaría el número de donaciones.

Hace veinte años que llevamos haciendo laparoscopia operatoria urológica (6), y queremos aprovechar para hacer hincapié en el hecho de que la cirugía asistida es de importancia decisiva, pero tiene sus indicaciones estrictas y precisas, que no deben sobrepasarse. En otras palabras, salvo en nefrectomías difíciles y poco más, la cirugía manualmente asistida no tiene razón de ser.

\section{CONCLUSIONES}

1. La utilización de un solo trocar disminuye considerablemente la agresión operatoria en comparación con la nefrectomía asistida convencional, lo que disminuye el dolor y acorta más si cabe el posto-peratorio.

2. La técnica descrita disminuye drásticamente el tiempo operatorio, incluso con respecto a la vía abierta convencional, ya que a menudo tardamos menos en quitar el riñón que en cerrar la punción y la brecha. 
3. La ligadura en bloque y a ciegas del pedículo no supone ningún riesgo añadido, porque con el dedo índice de la mano interna comprobamos la posición exacta de la grapadora antes de dispararla.

4. La posibilidad de fístula arteriovenosa es remota, porque la realidad es que con la grapadora, la arteria se deja junto a la vena, pero separada de la misma.

5. La técnica de momento ha demostrado su fiabilidad como cirugía oncológica, aunque nuestra estadística es limitada.

6. La mini-incisión debe ser lo más baja posible, por ser menos dolorosa, más estética y porque no hay posibilidad de lesionar los abdominogenitales.

7. El gasto en material laparoscópico es mínimo, puesto que sólo se requiere un trocar una grapadora y dos o tres cargas. Y, salvo excepciones, no se necesita ni aguja de Verres ni endotijera ni endodisector ni bolsas especiales ni endoclips ni ningún otro material laparoscópico.

8. Por todo ello, pensamos que debería plantearse su uso sistemático, incluso para la extracción en donante vivo.

\section{BIBLIOGRAFÍA Y LECTURAS}

\section{RECOMENDADAS (*lectura de interés $y$ ** lectura fundamental)}

**1. SÁNCHEZ DE BADAJOZ, E.; GOMÉZ DE LA CRUZ, J.; JIMÉNEZ GARRIDO, A. y cols.: "Cirugía vásculorrenal endoscópicamente asistida". Arch. Esp. Urol., 48: 25, 1995.

2. SÁNCHEZ DE BADAJOZ, E.: "Cirugía laparoscópica manualmente asistida: presente y futuro". Annals d'Urologia, 1: 27, 1995.

3. SÁNCHEZ DE BADAJOZ, E.; JIMÉNEZ GARRIDO, A.: "Laparoscopia manualmente asistida". Arch. Esp. Urol., 54: 349, 2001.

*4. SÁNCHEZ DE BADAJOZ, E.; JIMÉNEZ GARRIDO, A.: "Iniciación a la nefrectomía laparoscópica". Arch. Esp. Urol., 55: 425, 2002.

5. LÓPEZ CUBILLANA, P.; PRIETO GONZALEZ, A.; GÓMEZ GÓMEZ, A. y COLS.: "Nefrectomía radical manualmente asistida versus nefrectomía abierta en el tratamiento del carcinoma renal clínicamente localizado". Arch. Esp. Urol., 57: 833, 2004.

6. SÁNCHEZ DE BADAJOZ, E.; DÍAZ RAMÍREZ, F.; MARÍN MARTÍN, J.: "Tratamiento endoscópico del varicocele". Arch. Esp. Urol., 41: $15,1988$. 